\title{
The Accompanying Changes in Brain Structure of a Remitted Depression Patient with the Bupropion Treatment
}

\author{
Yi-Cheng Hou', Chien-Han Lai ${ }^{2,3}$ \\ 'Department of Nutrition, Taipei Tzu Chi Hospital, Buddhist Tzu Chi Medical Foundation, New Taipei City, ${ }^{2}$ Department of Psychiatry, Cheng \\ Hsin General Hospital, Taipei City, ${ }^{3}$ Department of Biomedical Imaging and Radiological Sciences, National Yang-Ming University, Taipei \\ City, Taiwan
}

The impacts from the bupropion on the brain structures have seldom been mentioned in the literature. The bupropion is a kind of antidepressant with dual action in the norepinephrine and dopamine receptors. Here we have a case to share about the bupropion-related effects in the brain structure.

KEY WORDS: Bupropion; Brain volume; Gray matter volume; White matter volume.

\section{INTRODUCTION}

The effects of the antidepressant bupropion on brain structures have seldom been reported. Bupropion has a dual action on norepinephrine and dopamine receptors. Here, we report a case showing bupropion-related effects on the brain structure.

\section{CASE}

A woman visited our clinic, who is a first-episode medication-naïve depression patient with a three-month history of depressed mood, suicidal ideation, lack of energy, feelings of worthlessness, psychomotor agitation, and significantly impaired occupational function (Hamilton Rating Scale for Depression [HRSD] score of 29). She had no other psychiatric or medical co-morbidities. Bupropion treatment $(150 \mathrm{mg} /$ day $)$ was initiated on her first visit to my clinic; her depression symptoms responded in the second week (bupropion $300 \mathrm{mg} /$ day; HRSD score 19) and she entered remission at six weeks (bupropion 300 mg/day; HRSD score 7). Her social and occupational function significantly improved (HRSD work and activ-

\footnotetext{
Received: February 23, 2015 / Revised: March 24, 2015

Accepted: April 6, 2015

Address for correspondence: Chien-Han Lai, MD

Department of Psychiatry, Cheng Hsin General Hospital, No.45,

Cheng Hsin St., Pai-Tou Destrict, Taipei City, Taiwan

Tel: +886-2-28264400 ext 3502, Fax: +886-2-28264570

E-mail: stephenlai99@gmail.com
}

ities item scores $4 \rightarrow 1$ ). No significant side effects or body weight changes were noted. She underwent three-dimensional fast spoiled gradient-echo recovery T1-weighted magnetic resonance imaging at baseline and six weeks after treatment initiation on a 3T Siemens scanner. We used the SIENA function (Structural Image Evaluation, using Normalization, of Atrophy) of the FMRIB software library toolbox (Oxford University) to analyze brain changes at a single time point ("cross-sectional", brain volume at baseline and six weeks) and two time points ("longitudinal", percentage brain volume change [PBVC]). The estimated PBVC (0.20968) represented a significant increase in brain volume upon remission in this depression patient. Normalized gray matter, white matter, and total brain volumes all increased after six weeks of bupropion treatment (Table 1).

\section{DISCUSSION}

The dopaminergic system is correlated with increased gray matter volume in the dorsolateral prefrontal cortex and striatal regions, which are associated with creativity. ${ }^{1)}$ The antidepressant bupropion inhibits dopamine and norepinephrine reuptake. Buproprion led to significant improvement in our patient's depression symptoms and significant increases in gray matter, white matter, and total brain volumes. The dopamine and norepinephrine agonist methamphetamine can also increase gray matter volume in the right putamen, which is associated with better in-

(a) This is an Open-Access article distributed under the terms of the Creative Commons Attribution Non-Commercial License (http://creativecommons.org/licenses/by-nc/4.0) which permits unrestricted non-commercial use, distribution, and reproduction in any medium, provided the original work is properly cited. 
Table 1. The changes in three volumes of the remitted depression patient with bupropion treatment for 6 weeks

\begin{tabular}{lrr}
\hline \multicolumn{1}{c}{ Volume $\left(\mathrm{mm}^{3}\right)$} & Baseline & \multicolumn{1}{c}{ 6th week } \\
\hline Gray matter volume & $863,375.21$ & $869,948.48$ \\
White matter volume & $812,055.43$ & $819,586.69$ \\
Total brain volume & $1,675,430.64$ & $1,689,535.17$ \\
\hline
\end{tabular}

hibitory control. ${ }^{2)}$ Apart from these dopamine-related mechanisms, bupropion probably also changes gray matter volume by preventing oxidative stress related to major depressive disorder, ${ }^{3)}$ or by modulating glutamate receptor function. ${ }^{4)}$ Gray matter volume might increase for several reasons: synaptic remodeling and neurogenesis; ${ }^{5)}$ stimulation of neurotrophic factors by antipsychotics; ${ }^{6)}$ prevention of oxidative stress or 6-OH-dopamine lesioning with subsequent increased glial cell proliferation in the frontal cortex; $;^{7)}$ or modulation of glutamate receptor function. $^{8)}$

One concern with these results is the variability of the SIENA method. A longitudinal survey comparing different segmentation methods found that SIENA gives large, heterogeneous values for brain volume changes, implying the variability of this method. ${ }^{9)}$ Another study mentioned that SIENA could use the outer skull surface for both time points to reduce the effects of scanner drift and inter-scanner variability on longitudinal morphometric results. ${ }^{10)}$ Therefore, our method still had some value for assessing longitudinal changes with bupropion treatment. This evidence of increased gray matter and total brain volumes with bupropion treatment has clinical implications for the possible effects of norepinephrine and dopamine reuptake inhibition on brain structure in the treatment of depression.

In conclusion, the treatment of depression with bupropion appears to be accompanied by changes in the gray matter, white matter, and total brain volumes.

\section{Acknowledgments}

This report was supported by the grant from the Cheng Hsin General Hospital and National Yang Ming University cooperative project 103F003C02.

\section{REFERENCES}

1. Takeuchi H, Taki Y, Sassa Y, Hashizume H, Sekiguchi A, Fukushima A, et al. Regional gray matter volume of dopaminergic system associate with creativity: evidence from voxel-based morphometry. Neuroimage 2010;51:578-585.

2. Groman SM, Morales AM, Lee B, London ED, Jentsch JD. Methamphetamine-induced increases in putamen gray matter associate with inhibitory control. Psychopharmacology (Berl) 2013;229:527-538.

3. Kornhuber J, Reichel M, Tripal P, Groemer TW, Henkel $\mathrm{AW}$, Mühle $\mathrm{C}$, et al. The role of ceramide in major depressive disorder. Eur Arch Psychiatry Clin Neurosci 2009;259 Suppl 2:S199-S204.

4. Witkin JM, Marek GJ, Johnson BG, Schoepp DD. Metabotropic glutamate receptors in the control of mood disorders. CNS Neurol Disord Drug Targets 2007;6:87-100.

5. Konradi C, Heckers S. Antipsychotic drugs and neuroplasticity: insights into the treatment and neurobiology of schizophrenia. Biol Psychiatry 2001;50:729-742.

6. Parikh V, Khan MM, Mahadik SP. Olanzapine counteracts reduction of brain-derived neurotrophic factor and TrkB receptors in rat hippocampus produced by haloperidol. Neurosci Lett 2004;356:135-139.

7. Wang HD, Dunnavant FD, Jarman T, Deutch AY. Effects of antipsychotic drugs on neurogenesis in the forebrain of the adult rat. Neuropsychopharmacology 2004;29:1230-1238.

8. Duncan GE, Miyamoto S, Leipzig JN, Lieberman JA. Comparison of the effects of clozapine, risperidone, and olanzapine on ketamine-induced alterations in regional brain metabolism. J Pharmacol Exp Ther 2000;293:8-14.

9. Durand-Dubief F, Belaroussi B, Armspach JP, Dufour M, Roggerone S, Vukusic S, et al. Reliability of longitudinal brain volume loss measurements between 2 sites in patients with multiple sclerosis: comparison of 7 quantification techniques. AJNR Am J Neuroradiol 2012;33:1918-1924.

10. Takao H, Hayashi N, Ohtomo K. Effect of scanner in longitudinal studies of brain volume changes. J Magn Reson Imaging 2011;34:438-444. 